\title{
My (Un)Favourite Things
}

\author{
Paweł Urzyczyn
}

Warsaw University

\begin{abstract}
The talk will be devoted to four open problems I was unsuccessfully trying to solve in the past. These problems concern:

- Regular and Context-Free Dynamic Logic;

- The question of polymorphic collapse;

- Higher-order push-down stores and procedures;

- Polymorphic definability of recursive data types.

Each of these problems addresses a basic issue in the logical foundations of computer science, and each has been open for a long time. This talk aims at bringing back the challenge, and sorting out the related confusions.
\end{abstract}

